\title{
Chondrosarcoma of Larynx: A Case Report with Review of Literature
}

\author{
${ }^{1}$ Rajesh Mundhe, ${ }^{2}$ Sagar Tarte, ${ }^{3}$ Prerana Patil, ${ }^{4}$ Majeda Malik, ${ }^{5}$ Salawa Bano, ${ }^{6}$ Roshan Chinoy
}

\section{ABSTRACT}

In spite of being the most common nonepithelial neoplasm, chondrosarcoma makes up only about $0.2 \%$ of all head and neck malignancies and approximately $1 \%$ of all laryngeal tumors. Primary laryngeal chondrosarcoma is rare with only 300 cases being reported in literature.

Herein, we present a case of 61-year old male who presented with long-standing neck mass. Histopathological examination, revealed it to be a chondrosarcoma arising from cricoid cartilage of larynx. Microscopic features with differential diagnoses and review of literature is being discussed.

Keywords: Cartilage, Chondrosarcoma, Cricoid, Larynx.

How to cite this article: Mundhe R, Tarte S, Patil P, Malik M, Bano S, Chinoy R. Chondrosarcoma of Larynx: A Case Report with Review of Literature. Int J Otorhinolaryngol Clin 2015;7(2): 72-74.

\section{Source of support: Nil}

Conflict of interest: None

\section{INTRODUCTION}

Laryngeal chondrosarcoma is rare with only 300 cases being reported in the literature. ${ }^{2}$ Most of these tumors present as a painless, slow growing mass in the anterior part of neck. These tumors are usually diagnosed on radiology. Histologically, these are low-grade tumors with a good prognosis, but at times one may get dedifferentiated areas. Conservative surgery is the primary treatment of choice with limited role of adjuvant chemotherapy or radiotherapy.

\section{CASE HISTORY}

A 61-year-old male, presented with a slow growing swelling located in the anterior part of the neck since 9 years. Initially, he was symptomless so, he sought no medical attention. Later, he developed increase in size of the swelling with hoarseness of voice and increasing difficulty in breathing over last 4 months.

\footnotetext{
${ }^{1}$ Consultant and Histopathologist, ${ }^{2-5}$ DNB Resident

${ }^{6}$ Head (Histopathology)

${ }^{1-6}$ Department of Pathology, Prince Aly Khan Hospital, Mumbai Maharashtra, India
}

Corresponding Author: Rajesh Mundhe, Consultant and Histopathologist, Department of Pathology, Prince Aly Khan Hospital Nesbit Road, Aga Hall, Mazagaon, Mumbai, Maharashtra, India Phone: 9833008599, e-mail: drrajeshmundhe@gmail.com
Computed tomography (CT) scan was done, and it showed a well defined, expansile, lobular, calcific/ossific mass measuring $3.6 \times 3.4 \times 2.4 \mathrm{~cm}$, along the posterior wall of the larynx, the mass was seen arising from the cricoid cartilage, extending superiorly till the level of false vocal cords and inferiorly up to 1st tracheal ring. There was no evidence of any neck adenopathy or vascular involvement (Fig. 1).

In view of the large mass obstructing, the laryngeal airway, total laryngectomy was performed and specimen was sent for histopathological examination.

Gross examination showed enlarged and distorted larynx with laryngeal orifice reduced to chink due to presence of large hard cartilaginous tumor. It measured $7 \times 6.5 \times 4 \mathrm{~cm}$ in size, and was seen involving the cricoids cartilage. The tumor was gritty, hard to cut with glistening, bluish translucent cut surface. Necrosis or fleshy areas were not seen (Fig. 2).

Microscopic examination showed a cellular cartilaginous tumor composed of chondrocytes with pleomorphic, hyperchromatic nuclei many of them showing prominent nucleoli (Figs 3A to C). Metaplastic bone formation was also noted in the tumor (Fig. 4). Dedifferentiated areas or necrosis was not seen. Lymphovasular emboli or soft tissue extension was not seen.

Final diagnosis of chondrosarcoma, grade II was offered.

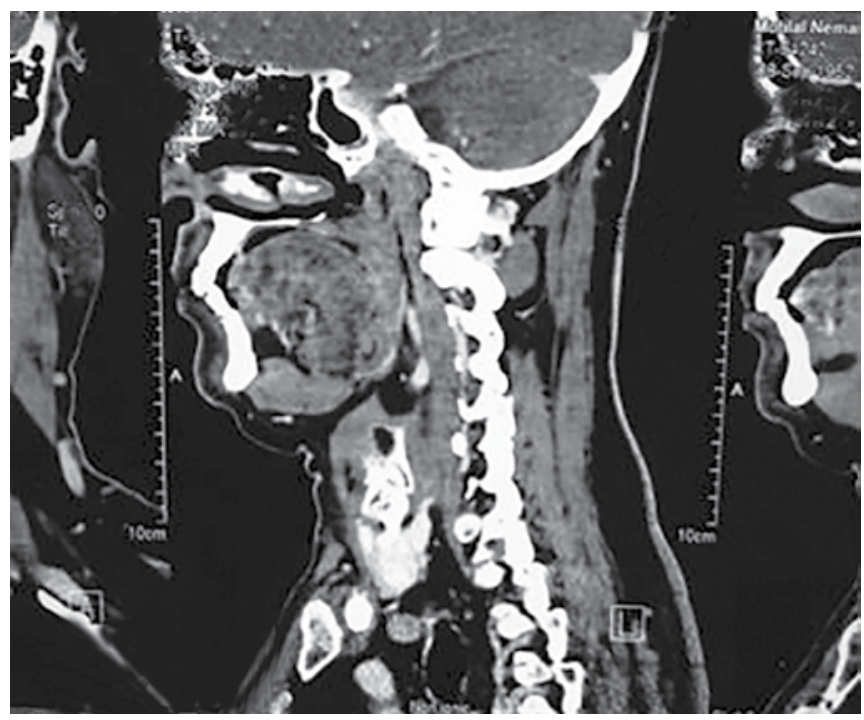

Fig. 1: Computed tomography scan showed a mass lesion in the larynx with areas of calcification 


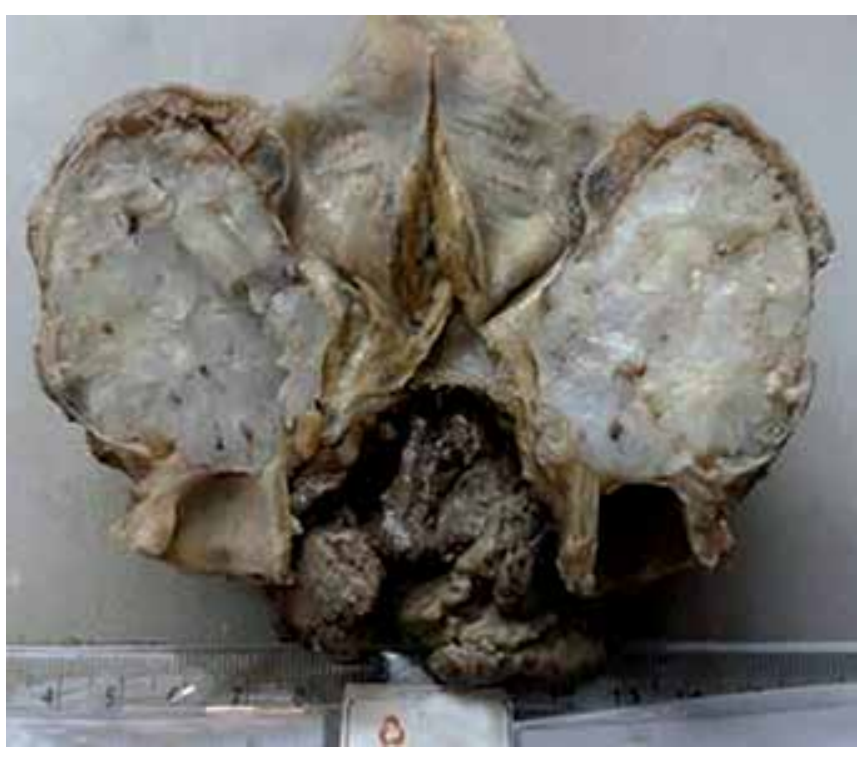

Fig. 2: Gross examination of the laryngectomy specimen showed glistening, translucent, bluish tumor involving the posterior wall with a stretched overlying mucosa

\section{DISCUSSION}

The cartilaginous tumors of larynx are rare and represent less than $1 \%$ of all tumors. These occur most often in men (sex ratio: 5/1), between 50 and 70 years, with an average age of 66 years. ${ }^{3}$ The preferred site in the larynx is cricoid cartilage $(75 \%)$ followed by the thyroid cartilage (17\%), the arytenoid $(5 \%)$ and epiglottis $(2 \%)^{3}$

The clinical symptoms are often gradual, polymorphic. The disease can remain asymptomatic for a long-time. Painless palpable lump is the most common presenting symptom followed by dyspnea, dysphagia and dysphonia. Other rarer clinical symptoms include stridor and sleep apnea.

The etiology of laryngeal chondrosarcoma is unknown, although it is assumed to derive from disordered ossification of the laryngeal cartilage. ${ }^{4}$

Whether identified on plain films or CT examinations, coarse or stippled calcification within a tumor mass is probably the single most helpful radiographic feature to correctly identify a laryngeal cartilaginous neoplasm (not necessarily separating a chondroma from a chondrosarcoma). ${ }^{1}$

Chondrosarcoma almost always arises from hyaline cartilages, i.e. cricoids, thyroid and arytenoids. Their occurrence in elastic cartilage is very rare. ${ }^{2}$

Majority of laryngeal chondrosarcoma are of lowgrade (grade I or II) on histology.

Identifying chondroid neoplasm on microscopy is not difficult; however, differentiating a low-grade chondrosarcoma (grade I and II) from benign chondroid tumors, like chondroma, chondromyxoid fibroma or chondrometaplasia would sometimes pose a diagnostic challenge.
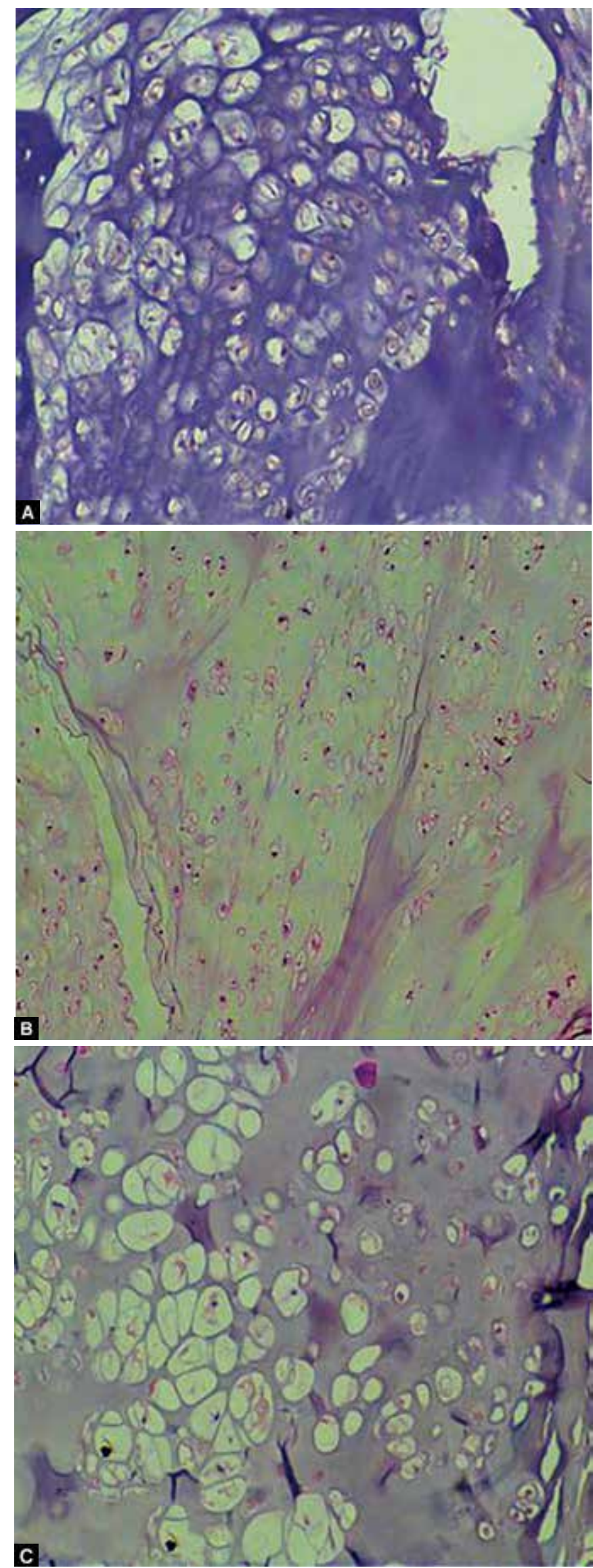

Figs 3A to C: Hematoxylin and eosin (H\&E) stained sections from the tumor show multiple cellular areas composed of chondrocytes with hyperchromatic nuclei and prominent nucleoli 


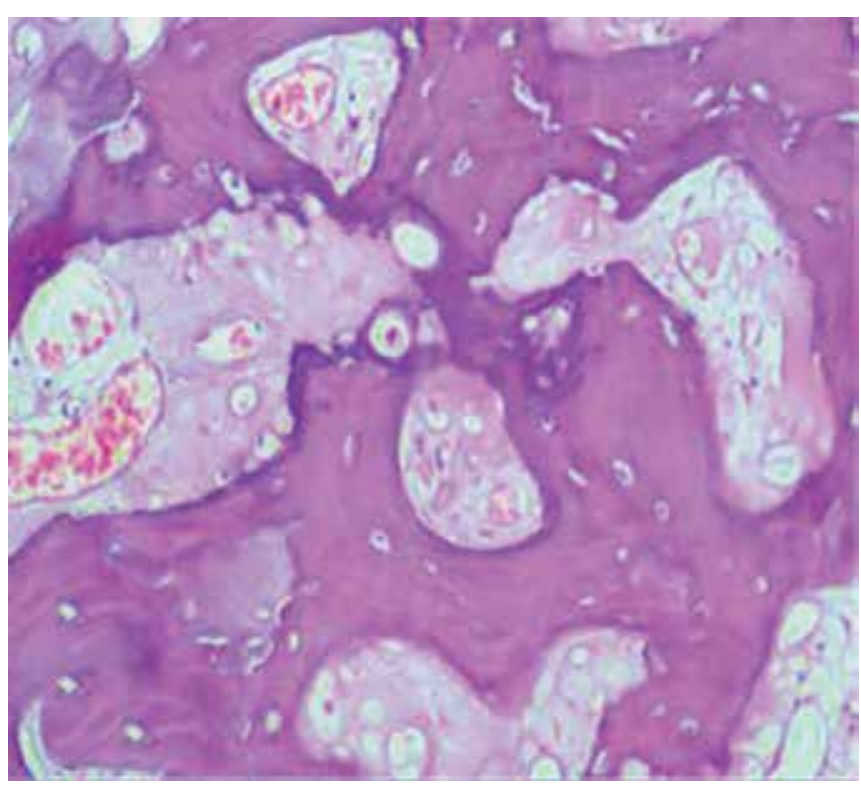

Fig. 4: Metaplastic bone formation seen in some areas within the tumor

It is difficult to distinguish between chondroma and a low-grade chondrosarcoma on histology alone. Chondromas of the laryngeal cartilages will resemble normal cartilage, nuclei will be uniform with single nuclei or rarely two. They will lack nuclear atypia, mitotic figures, and necrosis. Evidence of focal invasion goes in the favor of malignancy.

Chondrometaplasia of the larynx is usually located on the vocal cords. These lesions are usually $<1 \mathrm{~cm}$ in diameter, lack the characteristic lobular pattern of hyaline cartilage, and tend to be eosinophilic. The lack of nuclear atypia and infiltrative growth pattern will distinguish these lesions from chondrosarcomas.

Chondromyxoid fibroma usually occurs in long bones of a young adult. Microscopically, it shows myxochondroid lobules separated by intersecting bands of highly cellular tissue composed of fibroblast like spindle cells and osteoclasts. Occasionally, large pleomorphic cells are present, however, unlike in chondrosarcoma, mitoses are absent.

Grading is essential for chondrosarcoma as it predicts the histologic behavior and prognosis. Three tier grading system proposed by Evans et al is most widely accepted. ${ }^{5}$

Chondrosarcoma grading by Evans et $\mathrm{al}^{5}$ (Table 1).

Conservative surgery is the primary treatment of choice for laryngeal chondrosarcoma. Adjuvant therapy, radiation and chemotherapy, generally seems to be ineffective in the management of laryngeal chondrosarcoma as it is for chondrosarcomas in other anatomic sites.

Prognosis of this condition depends upon the radicality of the resection on the extension and histological
Table 1: Histological grading of chondrosarcoma as proposed by Evans et al

\begin{tabular}{|c|c|c|}
\hline Grade I & $\begin{array}{l}\text { Well-differentiated } \\
\text { (low-grade) }\end{array}$ & $\begin{array}{l}\text { Small, densely } \\
\text { staining nuclei often } \\
\text { with multiple nuclei } \\
\text { within one lacune }\end{array}$ \\
\hline Grade II & $\begin{array}{l}\text { Moderately differentiated } \\
\text { (intermediate grade) }\end{array}$ & $\begin{array}{l}\text { Increased cellularity, } \\
\text { significant amount } \\
\text { of cells having } \\
\text { moderately } \\
\text { sized nuclei, but } \\
\text { demonstrate a low } \\
\text { mitotic rate of less } \\
\text { than } 2 \text { mitoses } \\
\text { per HPF (also } \\
\text { includes myxoid } \\
\text { chondrosarcoma) }\end{array}$ \\
\hline Grade III & $\begin{array}{l}\text { Poorly differentiated } \\
\text { (high-grade) }\end{array}$ & $\begin{array}{l}\text { More than } 2 \text { mitoses/ } \\
\text { HPF, nuclear size } \\
\text { generally greater } \\
\text { than seen in grade II } \\
\text { (also includes } \\
\text { dedifferentiated } \\
\text { chondrosarcoma) }\end{array}$ \\
\hline
\end{tabular}

grade of the chondrosarcoma. Local recurrence is seen in approximately 20 to $60 \%$ of cases ranging from few months to several years after initial diagnosis. ${ }^{6}$

\section{CONCLUSION}

Chondrosarcoma rarely occurs in the larynx. It is usually of a lower-grade compared to those at the skeletal sites. It commonly arises from cricoid cartilage in posterior aspect. Surgery should be as conservative as possible. It rarely metastasizes and has a good prognosis.

\section{REFERENCES}

1. Thompson LD, Gannon FH. Chondrosarcoma of the larynx: a clinicopathologic study of 111 cases with a review of the literature. Am J Surg Pathol 2002;26(7):836-851.

2. Lewis JE, Barnes L, Tse LY, Hunt JL. Tumors of Bone and Cartilage. In: Barnes L, Eveson JW, Reichart P, Sindransky $\mathrm{D}$, editors. WHO classification of tumours in pathology and genetics of head and neck tumours. Lyon, Cedex, France, IARC Press: 2005. p. 158-159.

3. Berhill $\mathrm{CH}$, Toulba $\mathrm{H}$, Mezouri I, Zaidi H, et al. Laryngeal cahondrosarcoma: a case report with review of literature. Open Access Scientific Reports 2013;2(3):1-3.

4. Buda I, Hod R, Feinmesser R, Shvero J. Chondrosarcoma of larynx. Isreal Med Assoc J 2012;14(11):681-684.

5. Evans HL, Ayala AG, Romsdahl MM. Prognostic factors in chondrosarcoma of bone: a clinicopathologic analysis with emphasis on histologic grading. Cancer 1977;40(2):818-831.

6. Moerman M, Kreps B, Forsyth R. Laryngeal chondrosarcoma: an exceptional localization of a not unfrequent bone tumor. Sarcoma. 2009 (2009), Article ID 394908, 4 Pages. 